\title{
Community-Acquired Pseudomonas Aeruginosa Wrist Joint Arthritis in a Child - A Case Report
}

\author{
Manoj Kumar Jangid ${ }^{1}$ and Mohamad Ahmed Selim ${ }^{2}$ \\ ${ }^{1}$ Department of Paediatrics, NMC Royal Hospital, Dubai, United Arab Emirates \\ ${ }^{2}$ Department of Orthopaedics, NMC Royal Hospital, Dubai, United Arab Emirates
}

\section{Correspondence: \\ Manoj Kumar Jangid \\ Department of Paediatrics \\ NMC Royal Hospital \\ Dubai, United Arab Emirates \\ E-mail: drmanojjangid@gmail.com}

DOI: $10.3126 /$ jnps.v40i1.28854

Submitted on: $2019-05-08$

Accepted on: 2020-05-14

Acknowledgements: None

Funding: Nil

Conflict of Interest: None declared

Permission from IRB: Yes

\section{ABSTRACT}

Pseudomonas aeruginosa is a rare cause of bone and joint infection in an immunocompetent child. Rapid diagnosis and prompt therapy are required to prevent serious joint damage. We hereby present our experience with a case of septic arthritis involving distal radioulnar joint with associated distal radius osteomyelitis in a previously healthy six years old girl who presented with fever, swelling and pain of the right lower forearm. Pseudomonas aeruginosa was isolated from both joint fluid and blood. The child was successfully treated with a combination of surgical debridement and two weeks of intravenous and four weeks of oral antibiotic therapy.

Keywords: Pseudomonas aeruginosa; distal radioulnar joint; osteomyelitis; septic arthritis

To cite this article: Jangid MK, Selim MA. Community-Acquired Pseudomonas Aeruginosa Wrist Joint Arthritis in A Child - A Case Report. J Nepal Paediatr Soc. 2020;40(1):48-51.

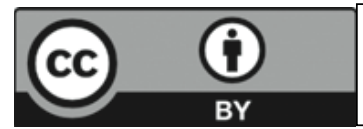

This work is licensed under creative common attribution 3.0 license 


\section{INTRODUCTION}

Septic arthritis of the wrist in children, though a rare occurrence, represents an orthopaedic emergency. Staphylococcus aureus is the usual offending microorganism at all ages. The infection usually occurs secondary to haematogenous spread from another site of infection, contiguous spread from adjacent tissue or by direct inoculation following puncture or trauma. ${ }^{1,2}$ Once an organism is seeded, cytokines and proteases are released which may ultimately lead to destruction of cartilage and if left untreated can result in adverse sequelae such as cartilage destruction, osteomyelitis, ankylosis, growth aberration due to physical damage, joint instability and restriction of movement. $^{2}$

We present a six years old girl with Pseudomonas wrist arthritis and associated distal radius osteomyelitis, who had no risk factors and was successfully managed with a combination of surgical debridement and antibiotic therapy.

\section{CASE REPORT}

A six years old, developmentally normal girl child with no significant past history, presented to our hospital with a history of worsening pain, swelling and redness of the lower end of right forearm for five days and fever for four days. During this period she was consulted with a local practitioner and was prescribed oral cefixime and ibuprofen. There was no history of trauma or any significant medical problem. On examination, there was

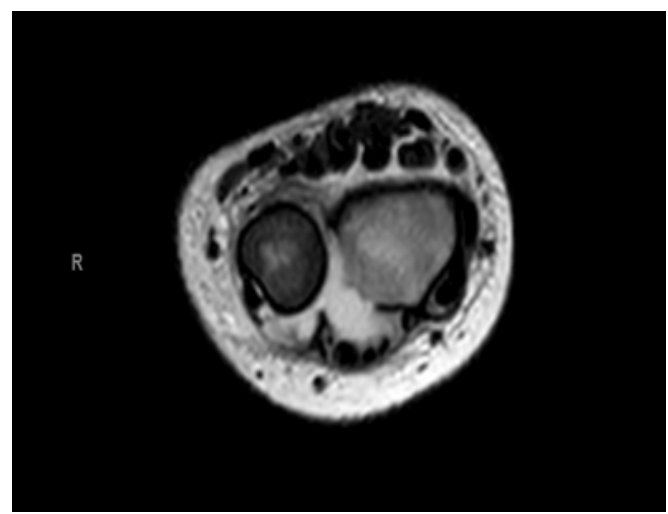

Figure 1. MR image of the distal forearm and wrist showing fluid collection in the right distal radioulnar joint and erosion along the distal metaphysis and epiphysis of the right radius swelling, erythema, warmth and tenderness of the lower end of right forearm, most prominent on dorsolateral side. The overlying skin did not show any skin trauma, bite marks, discharging sinuses or fluctuation. Systemic examination was all normal, including all other joints but she had dental caries. She was admitted for presumed bone and joint infection. Blood work up on admission revealed elevated inflammatory markers - C-reactive protein (CRP) $50 \mathrm{mg} / \mathrm{L}$ and erythrocyte sedimentation rate (ESR) $56 \mathrm{~mm} / 1^{\text {st }} \mathrm{hr}$. TLC was 15,000 cells $/ \mathrm{mm}^{3}$ and differential counts were also within normal limits. Blood culture was also collected. Plain radiograph of right forearm showed no abnormality. A consultation with orthopaedic team was made and intravenous ceftriaxone was started empirically.

Over the next two days, she continued to have fever, persistence of pain and swelling with rising CRP (71 mg/L). A magnetic resonance imaging (MRI) study demonstrated osteomyelitic changes in the lower end of radius with effusion in right distal radioulnar joint with surrounding soft tissue edema (Figure 1).

Blood culture sent on admission showed Pseudomonas aeruginosa growth, so antibiotics were changed as per the sensitivity report to Cefepime and Amikacin administered intravenously every 12 hourly. She underwent open irrigation and debridement where, purulent fluid was encountered in the joint, though the articular cartilage appeared to be in good condition. The pus

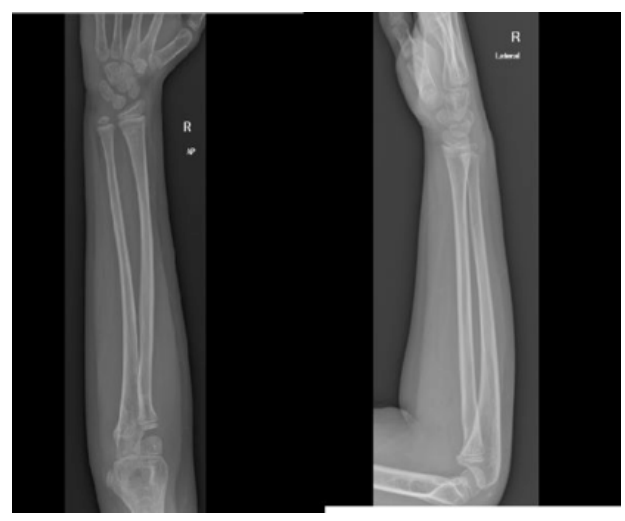

Figure 2. AP and lateral radiographs of forearm, one year after, showing complete recovery without any bony growth abnormalities 
culture of the joint also grew Pseudomonas aeruginosa. Postoperatively the child continued with IV antibiotics and splint immobilisation for a short time. Thereafter she remained stable and continued to improve with good relief of pain, reduction of swelling and improvement of movement of hand. The child was discharged after a total of two weeks of intravenous antibiotics and continued with oral ciprofloxacin at home for the next four weeks. The patient remained well, pain free with normal range of motion, without any sign of complications with normal radiograph at one year in follow up visits. (Figure 2)

\section{DISCUSSION}

Septic arthritis in children most commonly affects the lower extremity joints with knee, hip and ankle infections accounting for nearly 80 percent of cases. ${ }^{3}$ Septic arthritis of the wrist is a rare entity in children and four cases has been described so far in the literature. ${ }^{4-7}$ To the best of our knowledge this is the first reported case of acute septic arthritis of distal radioulnar joint due to a community acquired $P$. aeruginosa infection in a previously healthy child.

Classically, the diagnosis is made on the basis of history, physical examination, supportive laboratory studies and various imaging modalities. Septic arthritis commonly presents with fever and features of acute joint inflammation in the form of erythema, warmth and swelling with joint pain and/ or loss of function. Diagnosis of a septic wrist joint can be easily delayed in children especially in the absence of obvious penetrating trauma. In our child appropriate antibiotic therapy was delayed initially due to a sub acute presentation and later on there was a growth of unusual pathogen in blood culture -emphasising the importance of blood culture in such cases to make diagnostic and treatment decisions.

Most cases of septic arthritis are caused by haematogenous spread of bacterial pathogens while others can be caused from direct spread via trauma or infection from other neighbouring tissues. In our case the source of infection remained unclear though possibly a haematogenous spread from the dental caries could have led to the infection. It is well known that Pseudomonas aeruginosa is frequently present in periodontal infections and even a simple act of chewing can induce bacteremia. $^{8}$

Staphylococcus aureus remains the most common organism responsible for septic arthritis and osteomyelitis but our patient had $P$. aeruginosa infection. ${ }^{9}$ Patients developing $P$. aeruginosa joint infection usually have a history of prior traumatic events, hospitalisation, or chronic underlying disease but no such history was present in our case. ${ }^{10}$

Open surgical debridement is the preferred treatment and may be necessary depending on the extent of disease, response to antibiotic therapy and associated bone or soft tissue infections; though antibiotics for four to six weeks are generally sufficient in treating septic arthritis. With timely surgical debridement and an adequate course of antibiotics, our patient made a full recovery with complete resolution of clinical signs and symptoms with normalisation of inflammatory markers.

\section{CONCLUSIONS}

In conclusion, septic arthritis of wrist joint is a rare condition in children but should be considered seriously in all children with an acute onset of fever and features of acute joint inflammation such as swelling, pain or difficulty moving the wrist with elevated inflammatory parameters. Blood culture should always be sent in such cases as rare organisms like Pseudomonas can be the cause of septic arthritis in such instances and can be of paramount importance to have a good outcome.

\section{REFERENCES}

1. Rashkoff ES, Burkhalter WE, Mann RJ: Septic arthritis of the wrist. J Bone Joint Surg Am. 1983;65(6):824-828. 
2. Goldenberg DL: Septic arthritis. Lancet 1998; 351(9097):197-202.

3. Montgomery NI, Epps HR. Pediatric Septic Arthritis. Orthop Clin North Am. 2017;48(2):209-16. doi:10.1016/ j.ocl.2016.12.008

4. Wegner AM, Look N, Haus BM. Surgical Management of Multijoint Septic Arthritis due to Rat-Bite Fever in a Pediatric Patient: A Case Study. Case Rep Orthop. 2017; 2017:2183941. doi:10.1155/2017/2183941

5. Caksen H, Oztürk MK, Uzüm K, Yüksel S, Ustünbaş HB, Per H. Septic arthritis in childhood. Pediatr Int. 2000; 42(5):534-540. doi:10.1046/j.1442-200x.2000.01267.x

6. Rahman S, Salim F, Khandwala A: Septic arthritis of the hand and wrist in an 8-year-old girl not involving the radiocarpal joint. J Clin Case Rep. 2016,6:793. doi:10.4172/2165-7920.1000793

7. Silver J M, Hennrikus W. Septic Arthritis of the Pediatric Wrist: A Case Report and Review of the Literature. Cureus 12(3): e7444. doi:10.7759/cureus.7444

8. Pereira RS, Bonardi JP, Ferreira AC, Latini GL. An unusual case of dental infection by Pseudomonas aeruginosa causing a brain abscess: case report. Aust Dent J. 2017;62(4):523-7. doi: 10.1111/adj.12539.

9. McKay P, Formby P, Dickens JF, Gibson M. Osteomyelitis and septic arthritis of the hand and wrist. Curr Orthop Pract. 2010;21(6):542-50. doi: 10.1097/BCO.0b013e3181f1f2c1.

10. Richard F. Jacobs, Richard E. McCarthy, Joseph M. Elser. Pseudomonas Osteochondritis Complicating Puncture Wounds of the Foot in Children: A 10-Year Evaluation. J Infect Dis. 1989;160(4):657- 61. doi: https://doi.org/ 10.1093/infdis/160.4.657 